Application of Adaptive Beamforming to Signal Observations at the Mt. Meron Array, Israel

D. B. Harris

June 7, 2010 
This document was prepared as an account of work sponsored by an agency of the United States government. Neither the United States government nor Lawrence Livermore National Security, LLC, nor any of their employees makes any warranty, expressed or implied, or assumes any legal liability or responsibility for the accuracy, completeness, or usefulness of any information, apparatus, product, or process disclosed, or represents that its use would not infringe privately owned rights. Reference herein to any specific commercial product, process, or service by trade name, trademark, manufacturer, or otherwise does not necessarily constitute or imply its endorsement, recommendation, or favoring by the United States government or Lawrence Livermore National Security, LLC. The views and opinions of authors expressed herein do not necessarily state or reflect those of the United States government or Lawrence Livermore National Security, LLC, and shall not be used for advertising or product endorsement purposes.

This work performed under the auspices of the U.S. Department of Energy by Lawrence Livermore National Laboratory under Contract DE-AC52-07NA27344. 


\title{
Application of Adaptive Beamforming to Signal Observations at the Mt. Meron Array, Israel
}

\author{
D. B. Harris
}

The Mt. Meron array consists of 16 stations spanning an aperture of 3-4 kilometers in northern Israel. The array is situated in a region of substantial topographic relief, and is surrounded by settlements at close range (Figure 1). Consequently the level of noise at the array is high, which requires efforts at mitigation if distant regional events of moderate magnitude are to be observed. This note describes an initial application of two classic adaptive beamforming algorithms to data from the array to observe $\mathrm{P}$ waves from 5 events east of the array ranging in distance from 11002150 kilometers.

The two algorithms are approximate implementations of the classic adaptive beamformer proposed by Capon et al. (1967). This method forms a beam by shifting the sensor waveforms to align the incoming phase of interest (i.e. removing the propagation delays across the array for the target phase), applying FIR filters to each of the shifted sensor waveforms and summing the resulting filtered waveforms. The filters are designed by adjusting their coefficients to minimize the output power of the array subject to a constraint that the target phase is passed without distortion. The constraint is simple: the sum (over sensors) of the filters is an impulse function, zero except for one sample.

Exact design of the filters requires estimation of the covariance function for the background noise observed across the array. Under assumptions of stationarity, this is a matrix covariance function of the lag between observations at two different time instants. Design of the filters entails calculation of the inverse of this matrix function, which can be computationally complex. The two algorithms examined in this report are simpler approximations, more easily implemented.

The first algorithm is the method of Hisashi Kobayashi (1970), which finds the filter coefficients as the solution to a conjugate gradient optimization applied directly to the data, without the intermediate step of estimating the matrix covariance function from the data. The optimization objective is to minimize the total energy in the beam over a specified observation window. The algorithm can be more efficient because it usually is not allowed to iterate to conclusion. The beamforming results usually do not change substantially after a small number of iterations.

The second algorithm is the method of Otis Lamont Frost III (1972), which operates under the assumption that the structure of the ambient noise is not stationary, but varies slowly. The algorithm is time-adaptive in that it allows the coefficients to vary as a function of time (maintaining the fidelity constraint). It adjusts the coefficients using a simple gradient descent step with each time sample, where the gradient is estimated from a one-sample snapshot of the data covariance. This algorithm is attractive because it has the prospect of adapting to timevarying background noise, which is characteristic of the Mt. Meron array. It also is much faster than the Kobayashi algorithm and is suitable for continuous real-time operation. 
Both algorithms suffer from a potentially significant drawback: they assume that the incoming seismic waves are perfect plane waves, which means that the signals on each sensor are identical apart from propagation delays. The strongly heterogeneous seismic propagation medium guarantees that the plane-wave model is only approximately appropriate for seismic phases. The consequence of this fact is that these adaptive algorithms may suppress the desired signal as well as the noise. Two methods of mitigating this problem are usually tried. The first is to design the filters on a sample of pre-event noise and then apply them to the data window thought to contain the signal. This method was used successfully by Kobayashi in his 1970 paper, and a similar approach can be used with the Frost algorithm by employing a delay between the point of adaptation of the filters in the data stream and the application of the filters to detect the signal. This adaptation of the Frost algorithm was attempted in this study with negative results. Very poor noise rejection was obtained with any substantial delay.

The second approach is to use a very large observation window to design the filters that contains the signal. The expectation is that, for signals with very low SNR, the background noise power will dominate the objective function and the coefficient adaptation will not be driven to reject the signal. This approach appears to be largely successful with the Mt. Meron data, though some signal loss does occur. The tradeoff between noise suppression and signal loss is resolved in favor of noise rejection, leading to useful results for signals at the threshold of observation with conventional beamforming.

In the rest of this report, adaptive beamforming results for both methods are contrasted with conventional shift-and-sum beamforming results for four events. The results support the claim that adaptive beamforming provides useful improvement in event interpretation in cases where the signals are at the threshold of observation at the Mt. Meron array.

Both algorithms are very sensitive to the selection of parameters, which are now described. The principal parameters are the number of coefficients used in the FIR filters and the frequency band in which the filters are designed and applied. For the Kobayashi algorithm, the additional critical parameters are the size of the training window and the number of iterations used in the conjugate gradient algorithm. For the Frost algorithm, the additional parameter is a scale factor controlling the rate of adaptation of the algorithm.

In both algorithms, the beam is obtained as a sum of filtered multichannel traces of the form:

$$
y[n]=\sum_{k=-L}^{L} \mathbf{w}^{T}[k] \mathbf{x}[n-k]
$$

Here the bold characters indicate multichannel quantities, requiring vector operations. Note that the total number of vector filter coefficients is $2 L+1$. If the number of array channels is $N$, then the total number of scalar coefficients is $(2 L+1) N$. In this study, $L=30$, and $N=15$. One of the array stations was offline during the recording interval that we examine. 
The frequency band used for design and application of the beamformers is $0.5-2.0 \mathrm{~Hz}$. This fairly low frequency band is required because of the relatively high attenuation of $\mathrm{P}$ phases in this tectonic region for paths with lengths in the far regional distance range.

For the Kobayashi algorithm, the training window is the same as the interval of application of the filters to the data: 30 minutes, and contains the target signal. This long training window is used to assure that the ambient noise dominates the design of the filter coefficients. The number of iterations of the conjugate gradient adaptation is set to 10 .

The Frost algorithm updates the filter coefficients using a recursion:

$$
\left.\mathbf{w}[k]\right|_{n+1}=\mathbf{P}\left(\left.\mathbf{w}[k]\right|_{n}-\mu y[n] \mathbf{x}[n-k]\right)+\mathbf{F}[k] \quad k=-L, \cdots, L
$$

With $\mathbf{F}[k]=\delta_{k 0} \mathbf{c} /{ }_{N}, \mathbf{c}$ is a vector of all ones of dimension $N$, and $\mathbf{P}=\mathbf{I}-\mathbf{c c}^{T} / \mathbf{c}^{T} \mathbf{c}$

Together, $\mathbf{P}$ and $\mathbf{F}$ enforce the fidelity constraint and are fixed. The only additional parameter subject to tuning is the parameter $\mu$ controlling adaptation rate. This value is set to 0.000000002 , which assures slow adaptation consistent with the desire to make the processor sensitive principally to noise by having a long integration time.

A map of the locations of the 5 events used to test the two adaptive beamforming algorithms and compare them to a conventional shift-and-sum beamformer is shown in Figure 2. The events are located in or near the Zagros mountains of Iran, between 1100 and 2150 kilometers east of the Mt. Meron array. They range between 4.4 and 4.8 in magnitude, and appear to be close to the detection threshold for events in this distance range. Certainly some of the signals are not visible at all in the filtered single-channel waveforms and only become apparent after beamforming.

\section{Event 1: 5 Jan 2008, 00:37:40 GMT, 26.9N 54.9E, mb 4.6}

This earthquake is located some 1997 kilometers from the Mt. Meron array, in the distance range where transition from regional to teleseismic propagation occurs. The phase velocity for beamforming is $9.0 \mathrm{~km} / \mathrm{sec}$ and the (great circle) back-azimuth is 104.7 degrees rotating east from north. This event, among the five, provides the most compelling case for the use of adaptive beamforming. The $\mathrm{P}$ phase is not apparent at all as a change in amplitude in the conventional beam (Figure 2), and only marginally as a change on waveform character (Figure 3 ). In the two adaptive beams, it is clear both as a change in amplitude and character (frequency content). The Frost beam performs particularly well in this case.

\section{Event 2: 5 Jan 2008, 08:07:54 GMT, 31.47N 49.37E, mb 4.5}

This earthquake is located 1326 kilometers from the array. At this regional distance, the beamforming phase velocity is $8.0 \mathrm{~km} / \mathrm{sec}$ (Pn propagation assumed). The back-azimuth is 93.6 degrees east of north. This event provides a second example where the use of adaptive 
beamforming methods results in a fairly substantial improvement in the interpretability of the event. In this case (see Figures 5 and 6), the Pn phase is not well observed in the conventional beam, but is apparent as an increase in waveform amplitude (Figure 5) and a change in frequency content (Figure 6) in the two adaptive beams. In this example, it is entirely possible that Pn would be missed altogether in an examination of the conventional beam (and certainly by an automatic power detector). It is likely to be detected in the adaptive beams.

\section{Event 3: 22 Mar 2008, 00:01:03 GMT, 28.27N 57.18E, mb 4.6}

This earthquake is located 2148 kilometers from the array. The assumed phase velocity is 9.0 $\mathrm{km} / \mathrm{sec}$ and the backazimuth is 98.37 degrees. Figure 7 shows the results of the beamforming algorithms for this event. In this case, the $\mathrm{P}$ arrival has a reasonably high SNR in the conventional beam, so that use of the adaptive algorithms is not essential. The SNR is improved in the Kobayashi beam: though the $\mathrm{P}$ phase experiences some loss, it appears that noise power is suppressed disproportionately. Noise suppression is not very great with the Frost algorithm, but the $\mathrm{P}$ signal is relatively unscathed.

\section{Event 4: 22 Mar 2008, 15:51:57 GMT, 33.46N 47.57E, mb 4.4}

This earthquake is situated 1134 kilometers from the array. The assumed phase velocity is 8.0 $\mathrm{km} / \mathrm{sec}$ and the backazimuth is 84.2 degrees. The results of beamforming are shown in Figures 8 and 9. The adaptive beams show marginal improvements in the visibility of the Pn which in this case might assist in identifying the presence of Pn before the larger and more obvious Pg phase. The Kobayashi algorithm performs better in this respect than the Frost algorithm. However, the improvement is not very significant for either adaptive algorithm in this example.

\section{Event 5: 23 Mar 2008, 12:11:32 GMT, 37.31N 48.51E, mb 4.8}

This earthquake is 1284 kilometers from the array. The assumed phase velocity is $8.0 \mathrm{~km} / \mathrm{sec}$ and the backazimuth is 64.6 degrees. The Pn signal from this event has a fairly high SNR in the conventional beam, hence the adaptive beams do not add much (see Figure 10). The background noise is suppressed slightly by the adaptive algorithms, but the signal experiences some loss as well (especially with the Kobayashi algorithm). The principal improvements apparent with the adaptive algorithms lie in the greater rejection of two transient signals that appear after the earthquake signal. Overall, the Frost algorithm appears to perform slightly better in this example. It is worth noting the substantial Pn signal loss (compared to the single channel shown) in the conventional beam. This is an indication of the degree to which the spatial structure of Pn departs from a plane wave.

In summary, adaptive beamforming appears to provide some value in event observation and interpretation for the Mt. Meron array for events close to the detection threshold in the farregional to near-teleseismic distance range. The two algorithms tested do not performance worse than conventional beamforming for any of the events tested, and perform crucially better for two events. The implementation of Frost's algorithm as tested appears to experience less signal loss than the Kobayashi algorithm, though Kobayashi's algorithm often has superior noise-rejection 
characteristics. Tests were performed in the $0.5-2.0 \mathrm{~Hz}$ band where propagation is best off to the east of the array. At higher frequencies (2-5 Hz), adaptive beamforming appears to offer no advantage, suggesting that noise is not spatially correlated above $2 \mathrm{~Hz}$ in an exploitable fashion. 


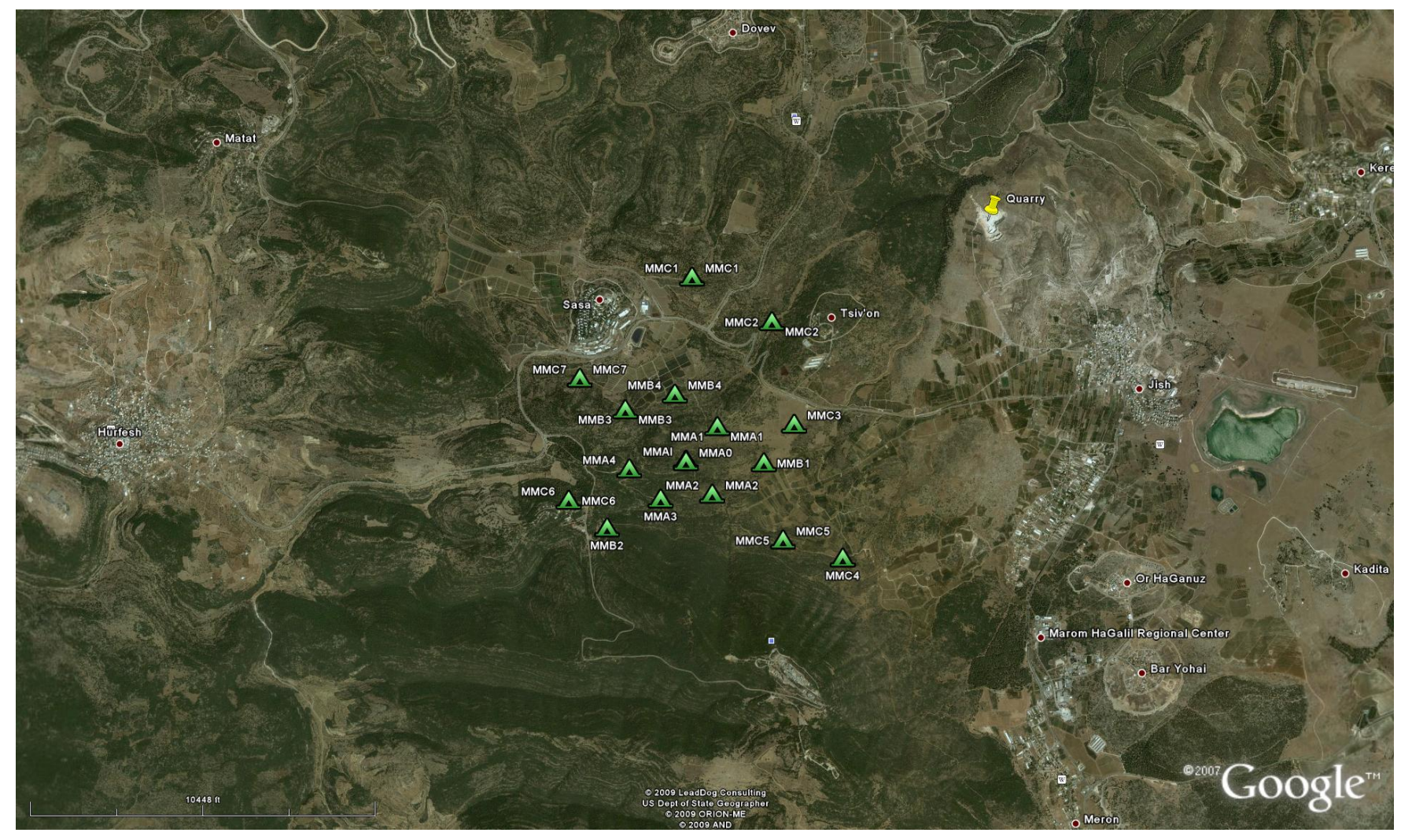

Figure 1 Satellite photograph of the Mt Meron array location showing proximity to settlements (greyish areas to the west and east). The sensor locations are indicated with triangular green symbols. Substantial topography is apparent. The road to Mt. Meron summit surrounds and traverses the array. 


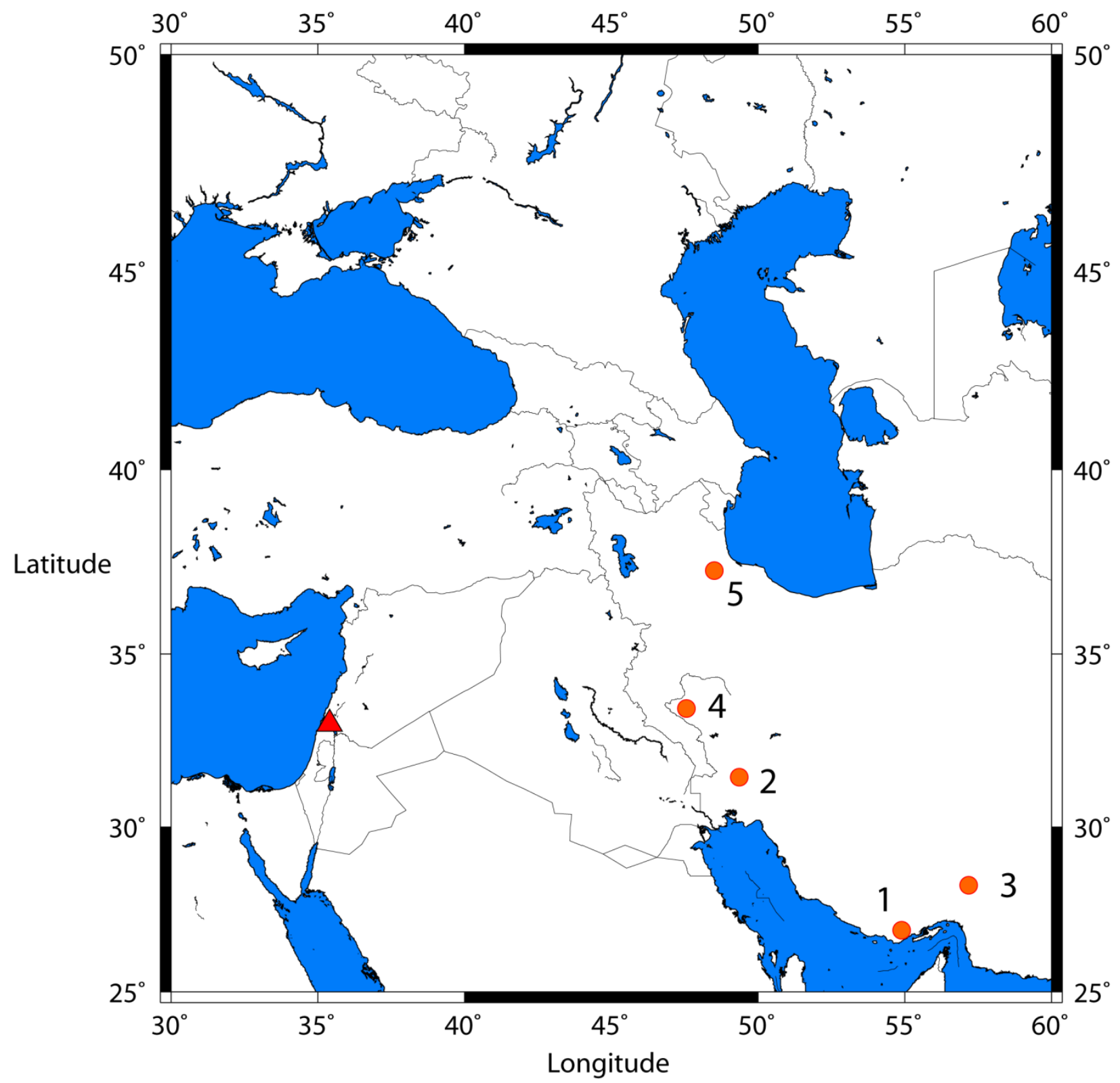

Figure 2 Locations of the Mt Meron array (red triangle) and the 5 events (orange circles) used in this beamforming study. 


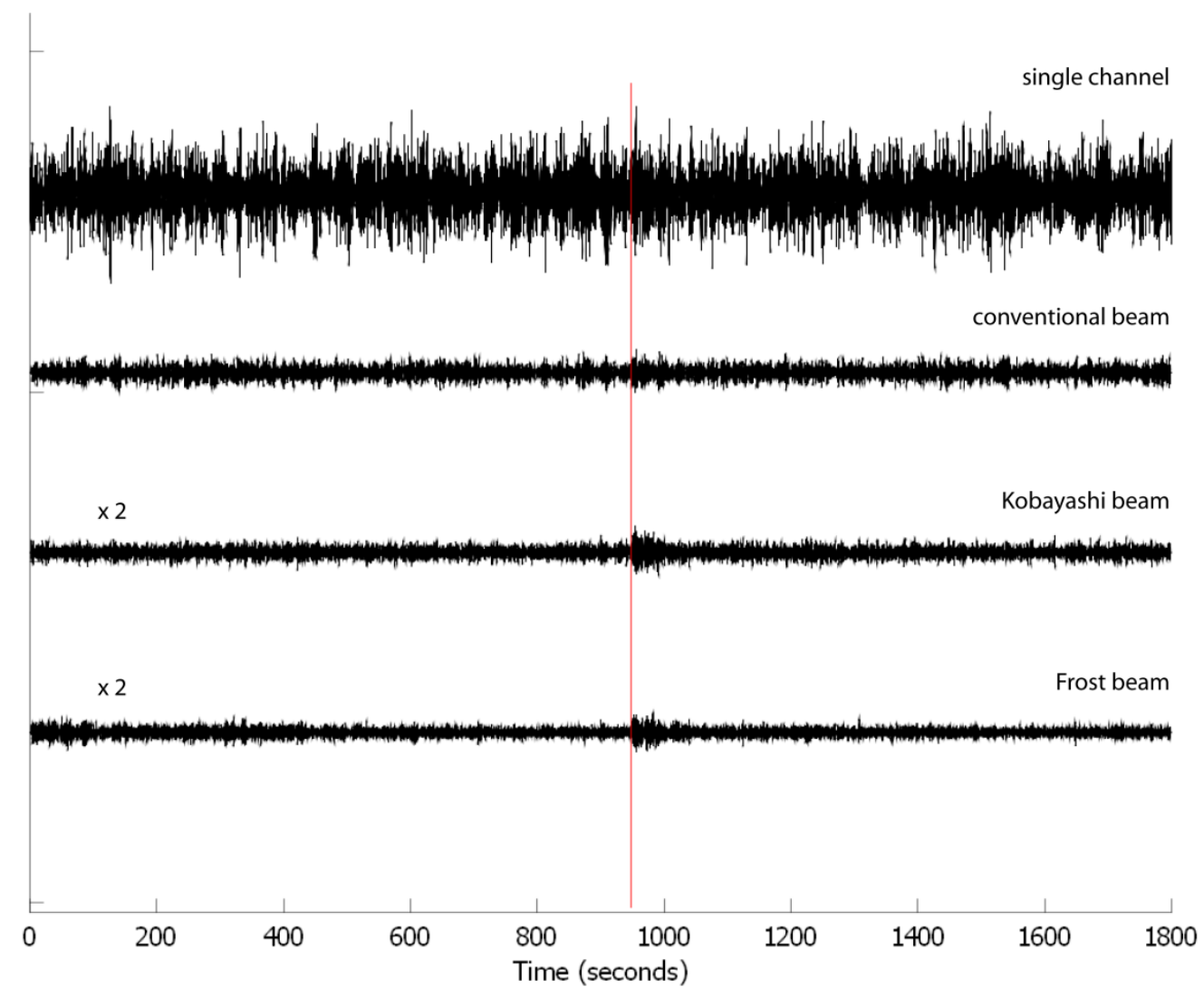

Figure 3 Waveforms from event 1 demonstrate that adaptive beamforming can provide an interpretable signal when the signal in a conventional beam is at or below the threshold of observation. The top trace is a single channel from the array filtered into the detection band of $0.5-2.0 \mathrm{~Hz}$. The second trace is the conventional beam, which shows no signal at this scale. The bottom two traces display the two adaptive beams and have been scaled by a factor of 2 to improve the visibility of the $\mathrm{P}$ phases. The $\mathrm{P}$ arrival time predicted by AK135 is indicated by the red vertical line. Detail of the $P$ phases is shown in the next figure. 


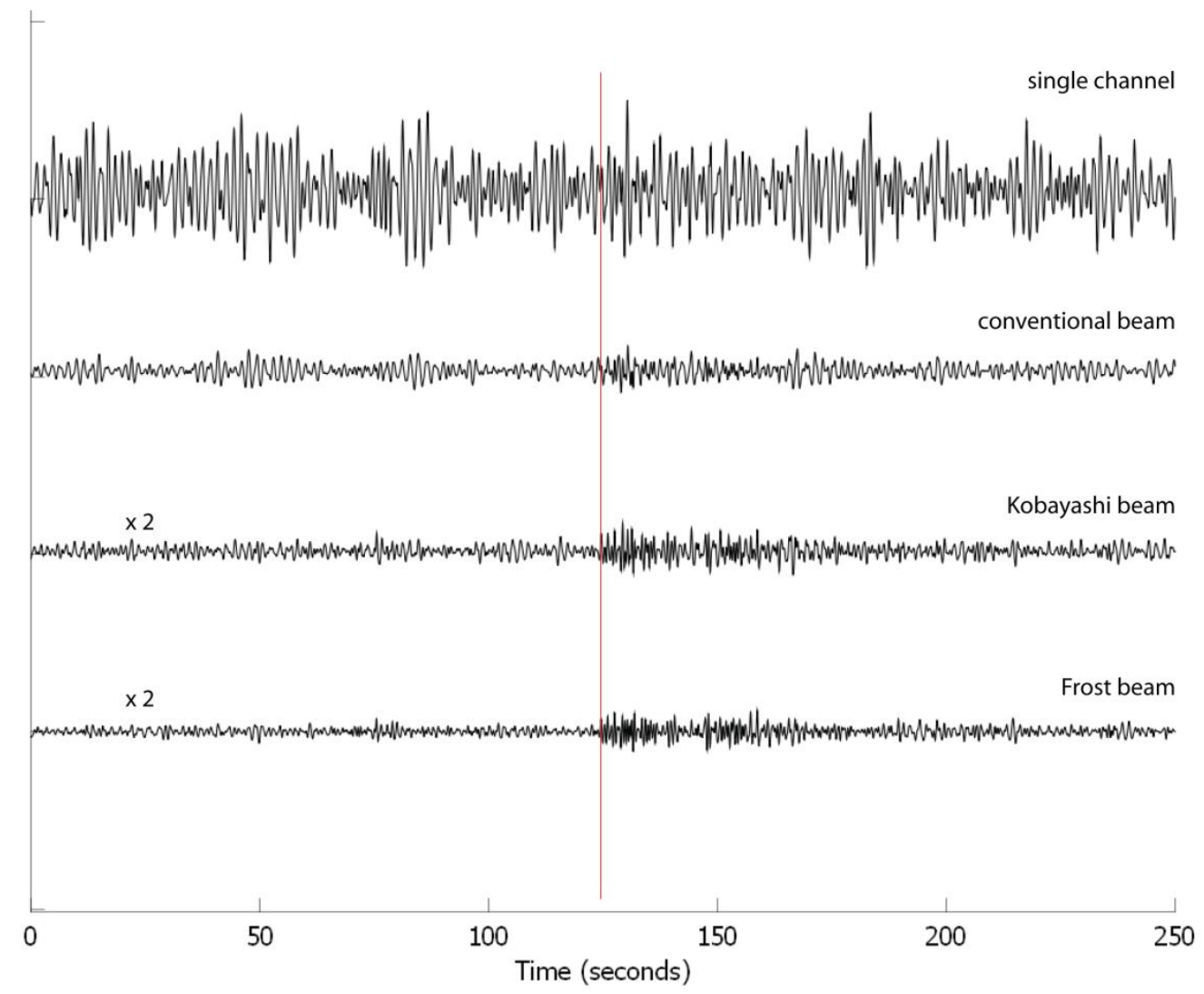

Figure 4 Detail of the $P$ phase estimates made with conventional beamforming (second trace) and the adaptive beamformers (bottom 2 traces, scaled by a factor of 2). The Frost beamformer performs particularly well in this example, producing a $\mathrm{P}$ phase observation that is readily picked. The red vertical line is the $P$ arrival time predicted by AK135. 


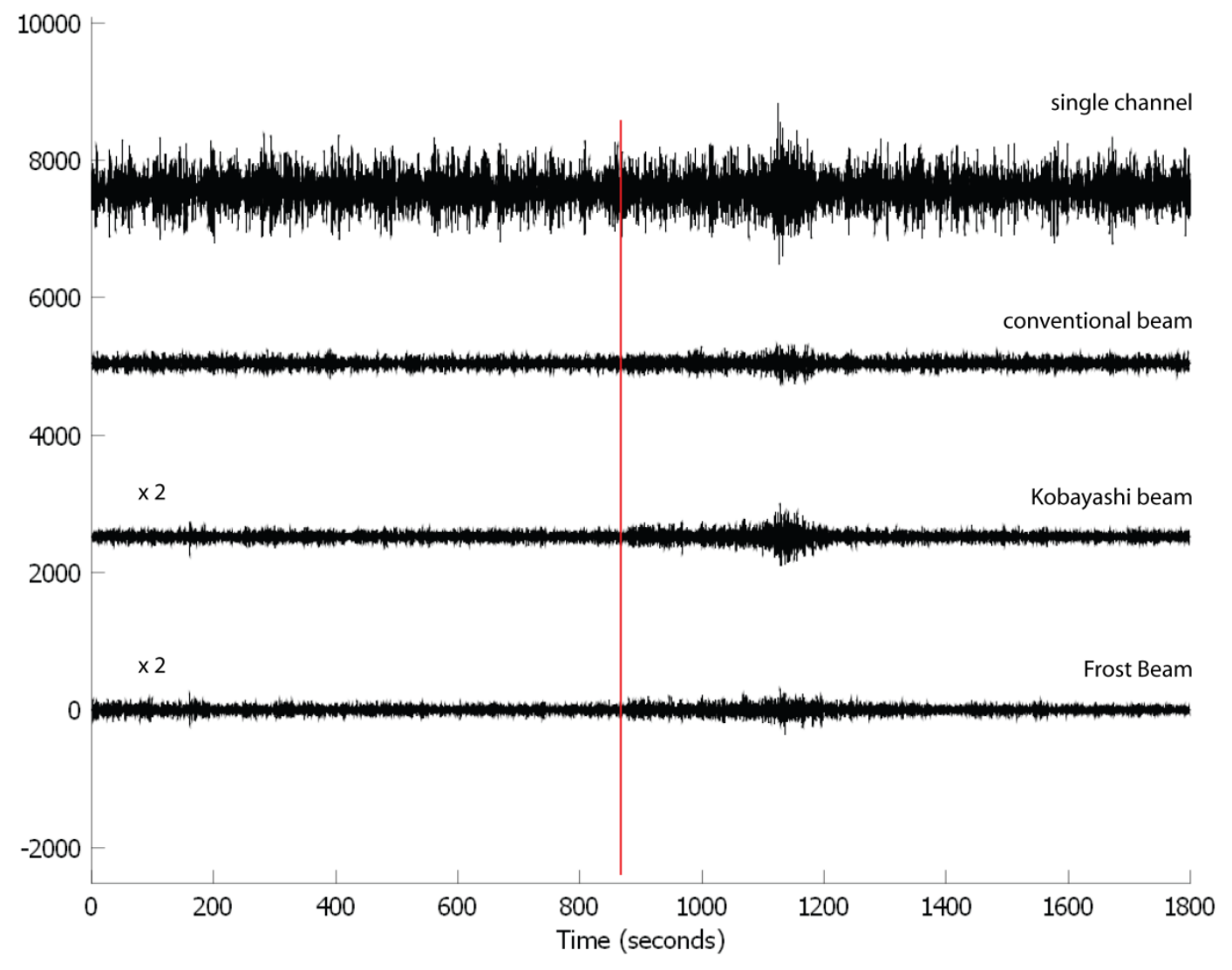

Figure 5 Comparison of beams for event 2. The red line indicates the predicted Pn time based upon a velocity of $8 \mathrm{~km} / \mathrm{sec}$ and no correction for the crustal legs of propagation. Consequently it appears a bit early. Detail of the $\mathrm{P}$ arrival is shown in the next figure. The bottom two beams are shown scaled by a factor of two. 


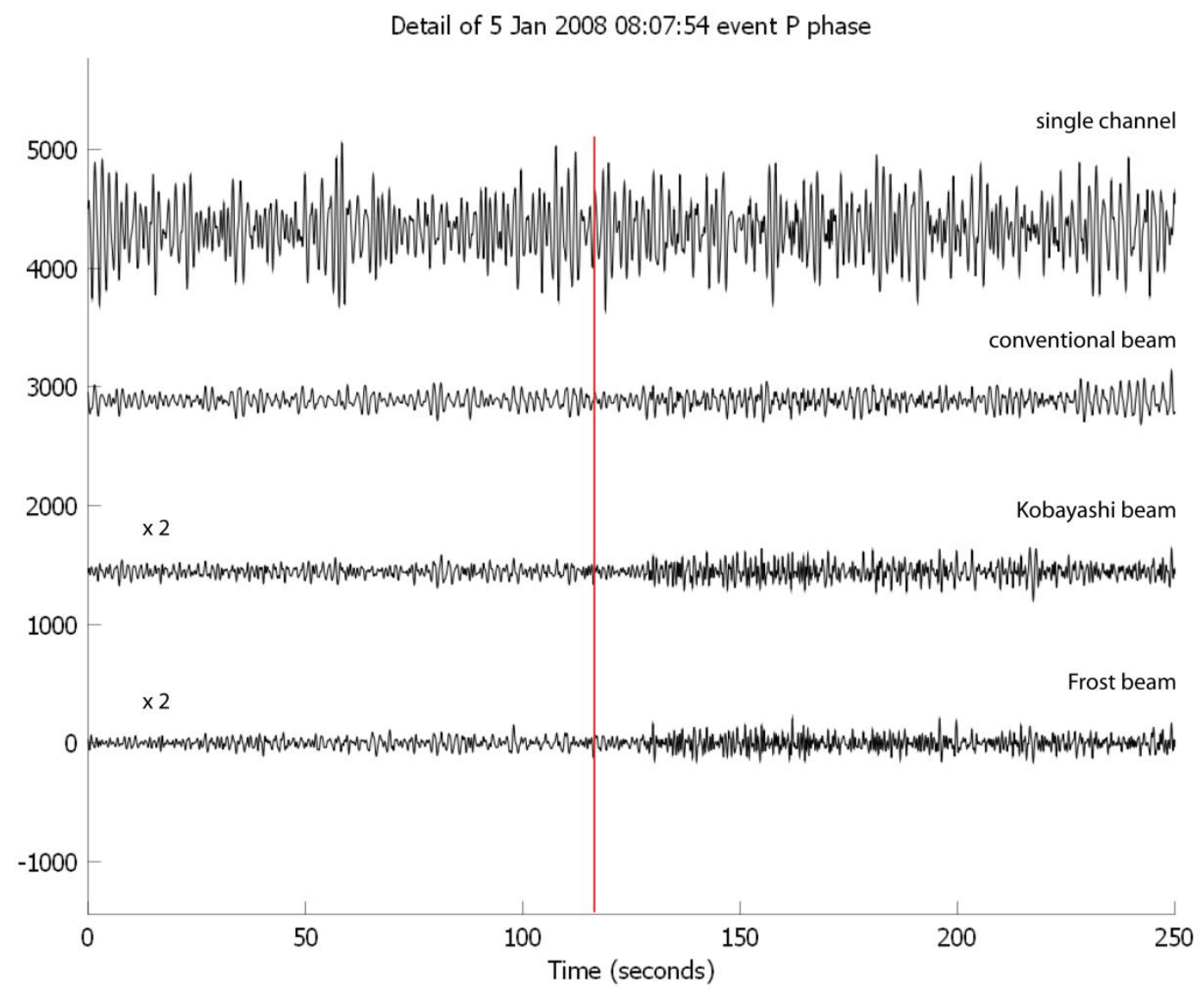

Figure 6 Detail of the P arrival for event number 2 . The conventional beam does not have a clearly interpretable arrival. The two adaptive beams have arrivals that may be picked within a few seconds. Again the red line indicates a predicted Pn arrival time without crustal leg corrections (so is early). The bottom two beams are shown scaled by a factor of two. 


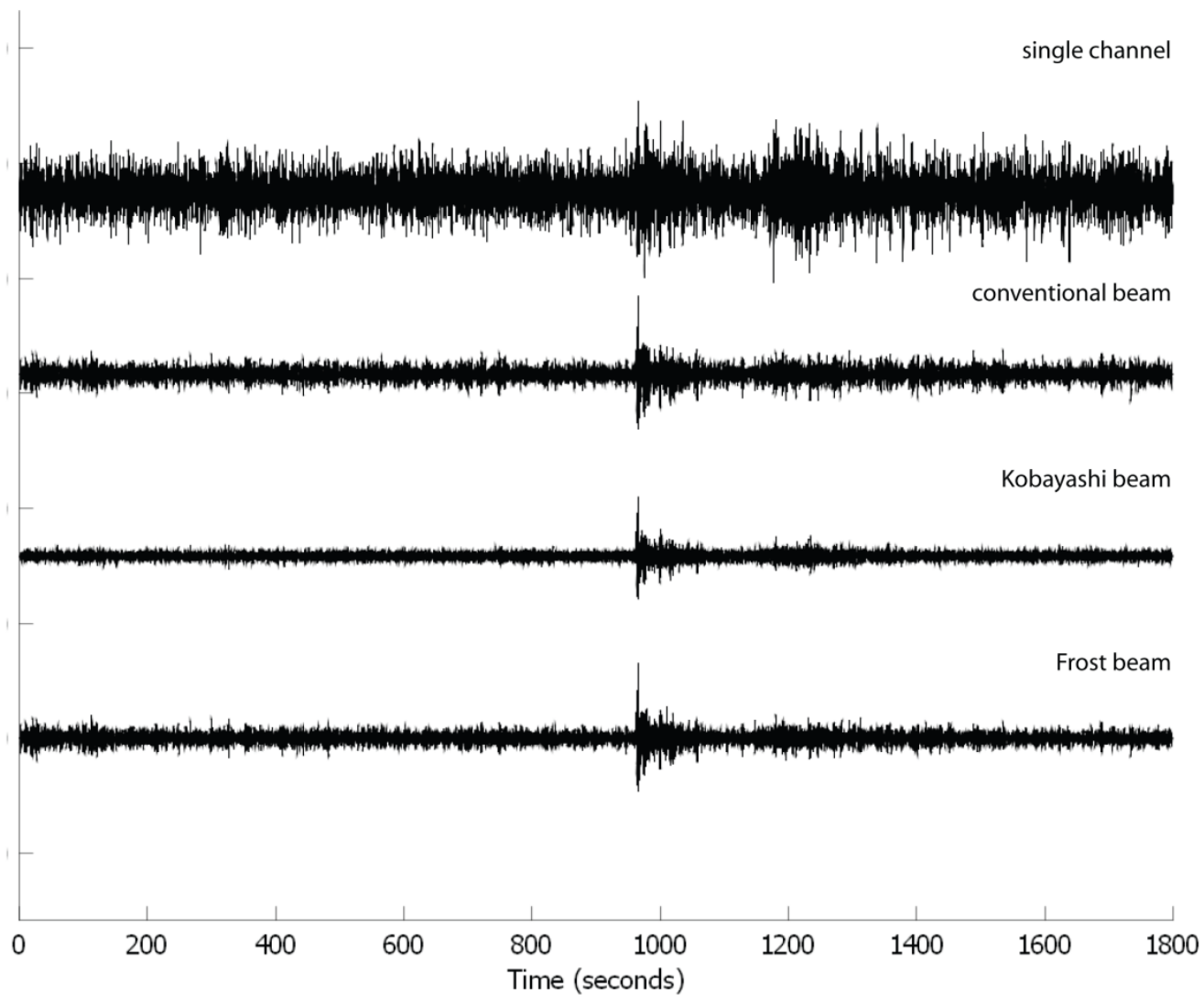

Figure 7 Beamforming results for event 3. In this case the conventional beam is adequate to pick the $P$ phase and adaptive beamforming is not necessary. The Kobayashi beam has lower background noise than the conventional beam, but the signal has been suppressed slightly. The net result is not a substantial increase in SNR. The Frost beam shows only marginal noise suppression, but little signal amplitude loss. The result is possibly slightly better than the conventional beam, but is not significant. 


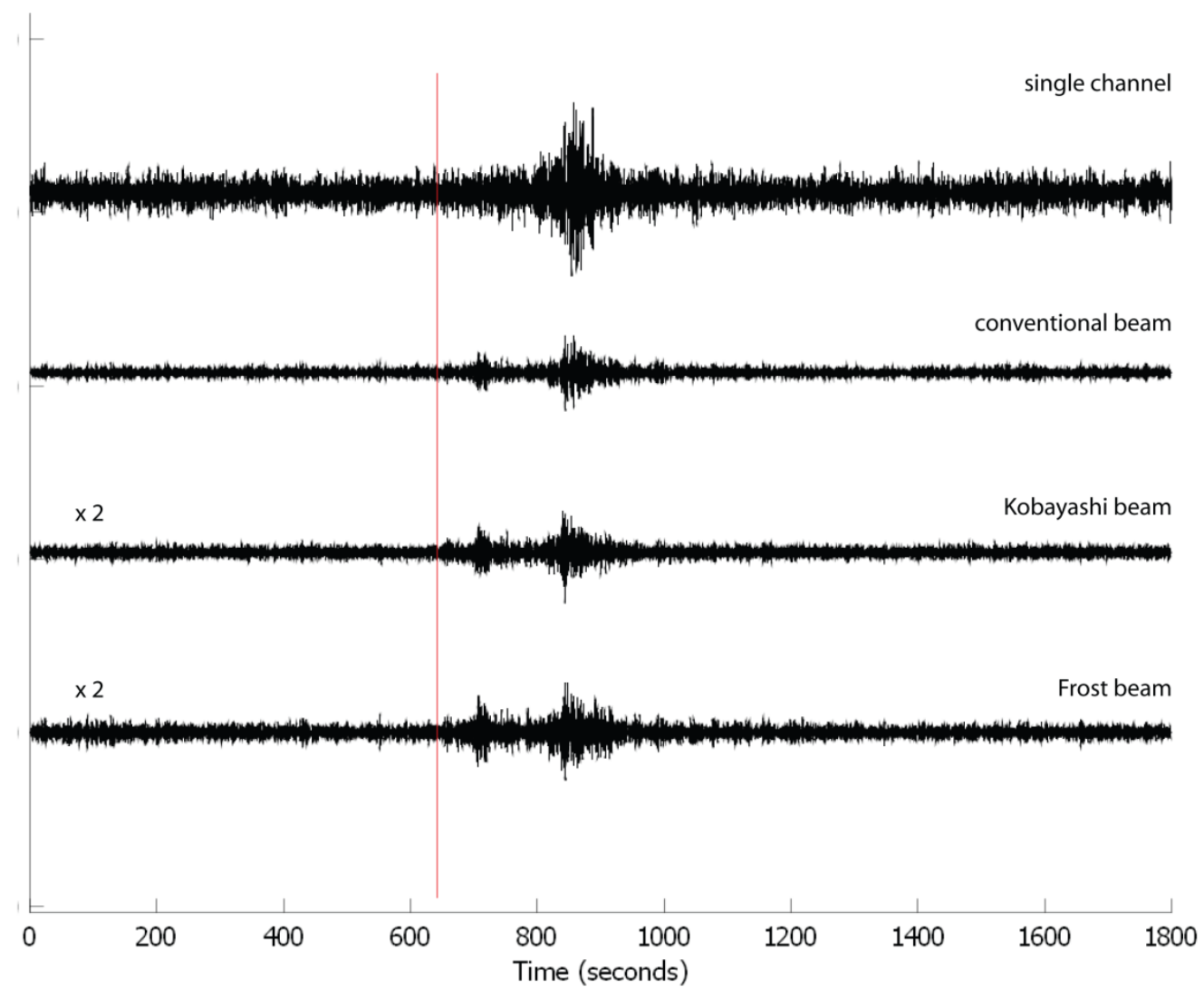

Figure 8 For event 4, the adaptive beams show a slight improvement in the ability to observe the $\mathrm{Pn}$ phase. The Kobayashi beam is slightly better in this respect at this scale than the Frost beam, due to more effective noise suppression. The two adaptive beams are shown here scaled by a factor of two to improve the visibility of the Pn arrival. The Pn arrival time predicted from the origin time, the distance and an assumed phase velocity of $8.0 \mathrm{~km} / \mathrm{sec}$ is indicated by the vertical red line. Detail of the $P$ phases is shown in the next figure. 


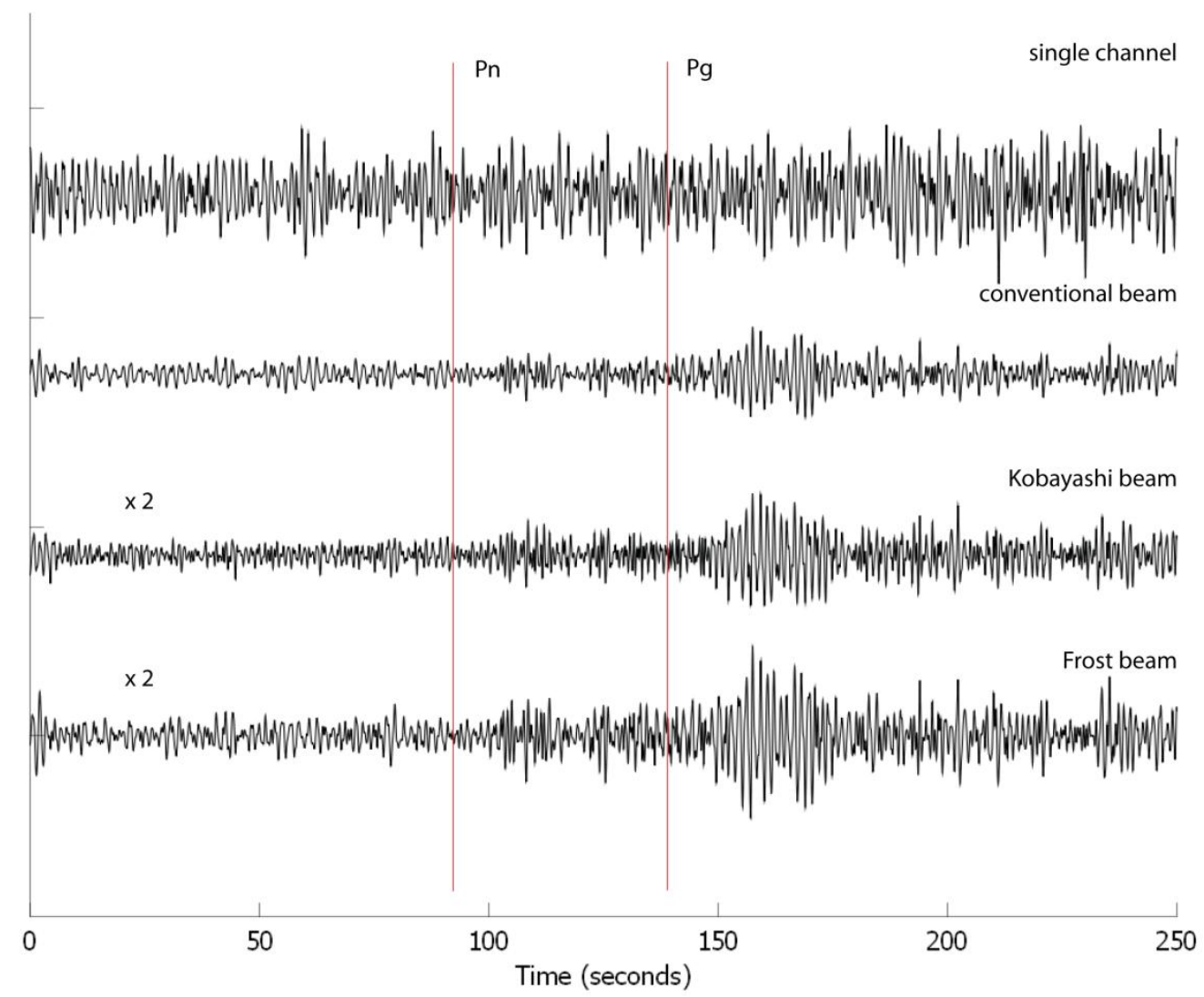

Figure 9 Detail of the $\mathrm{P}$ phases $(\mathrm{Pn}$ and $\mathrm{Pg}$ ) for event 4. The adaptive beams (shown here scaled by a factor of 2) show marginal improvement in the visibility of the Pn phase. In this case the SNR of the Pg phase appears also to have been increased slightly. Theoretical Pn and Pg arrival times based upon assumed phase velocities of $8.0 \mathrm{~km} / \mathrm{sec}$ and $6.0 \mathrm{~km} / \mathrm{sec}$ are shown by the vertical red lines. No crustal leg corrections are included in the predictions, so the predicted Pn time is early. 


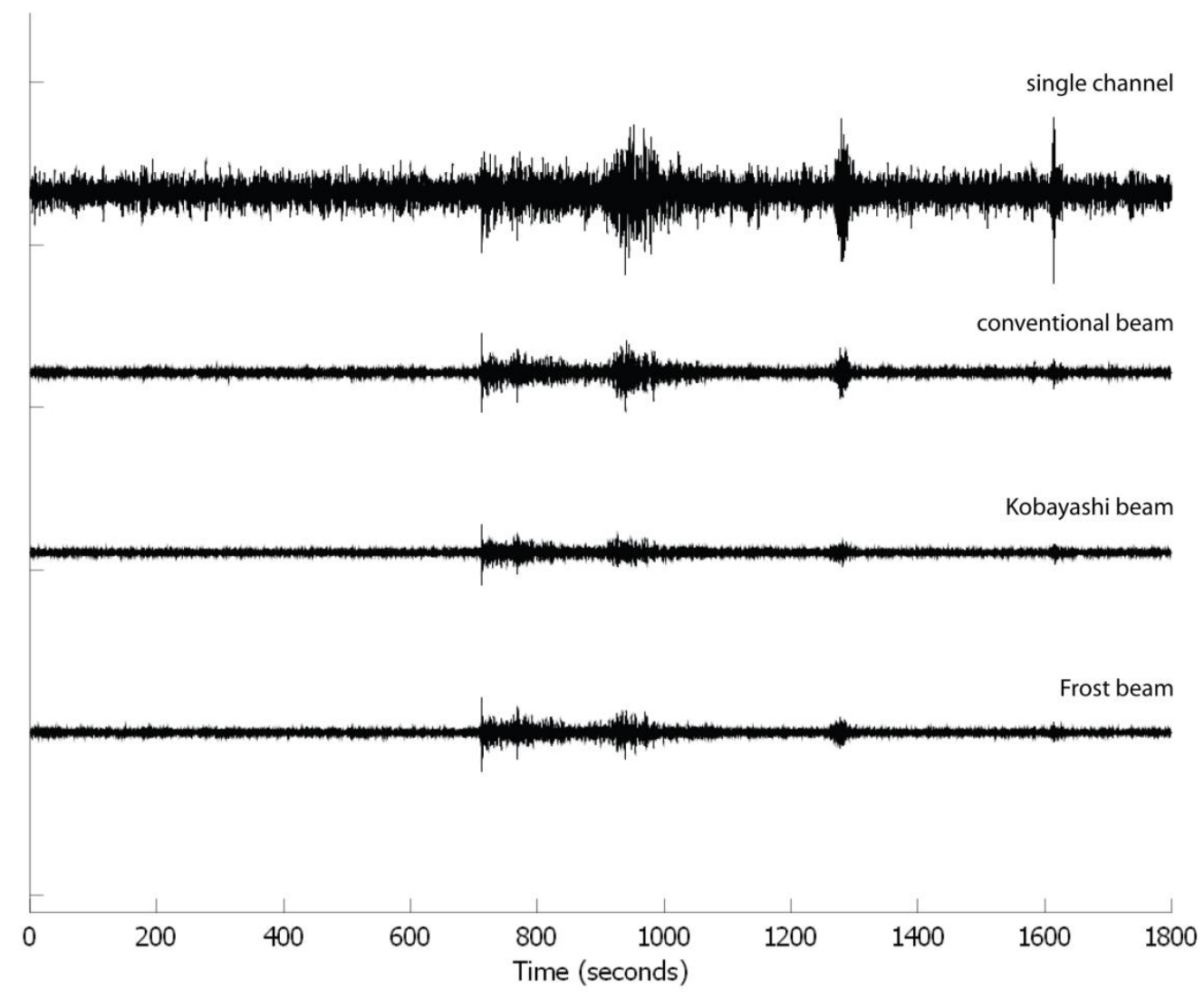

Figure 10 The three beams for event 5 do not show much improvement for the adaptive beamforming algorithms. While the Kobayashi beam has slightly lower background noise, the $P$ waveform is slightly suppressed as well. Suppression of the P phase is negligible with the Frost algorithm, but the noise is suppressed only slightly. The principal value of the adaptive beams in this example lies in the greater suppression of two later unrelated transients. 


\section{References}

Capon, J., R. J. Greenfield and R. J. Kolker (1967), Multidimensional maximum-likelihood processing of a large aperture seismic array, Proceedings of the IEEE, 55, 192-211.

Frost, O. L. (1972), An algorithm for linearly-constrained adaptive array processing, Proceedings of the IEEE, 60(8), 926-935.

Kobayashi, H. (1970), Iterative synthesis methods for a seismic array processor, IEEE Trans. on Geoscience Electronics, Vol. GE-8(3), 169-178. 